\title{
Green and Sustainable Process for the Recovery of Gold from Low Grade Sources Using Biogenic Cyanide Generated by Bacillus megaterium: A Comprehensive Study
}

\author{
Fariborz Faraji*, Jingxiu Wang, Harshit Mahandra, Ahmad Ghahreman* \\ Environmental and hydrometallurgy group at The Robert M. Buchan Department of Mining, \\ Queen's University, 25 Union Street, Kingston, ON, K7L 3N6, Canada \\ * Corresponding authors \\ E-mail address: \\ f.faraji@queensu.ca (Fariborz Faraji) \\ ahmad.g@queensu.ca (Ahmad Ghahreman)
}

Number of pages: 10

Number of figures: 4

Number of tables: 6

\footnotetext{
*Corresponding authors, E-mail address: f.faraji@queensu.ca (F. F.), ahmad.g @queensu.ca (A. G.).
} 


\section{Supporting figures:}
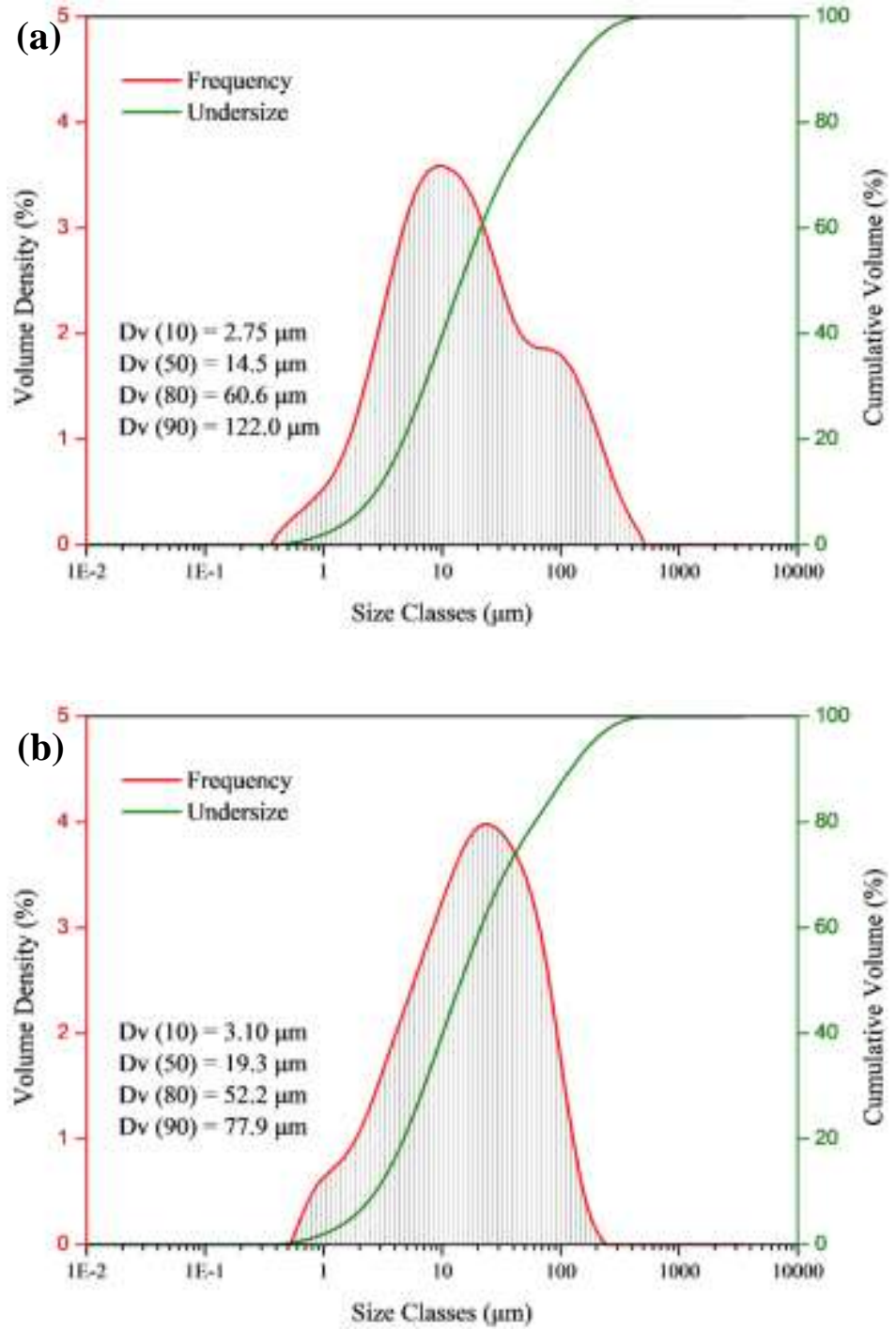

Figure S1. Particle size distribution of samples a) O1 and b) O2. 

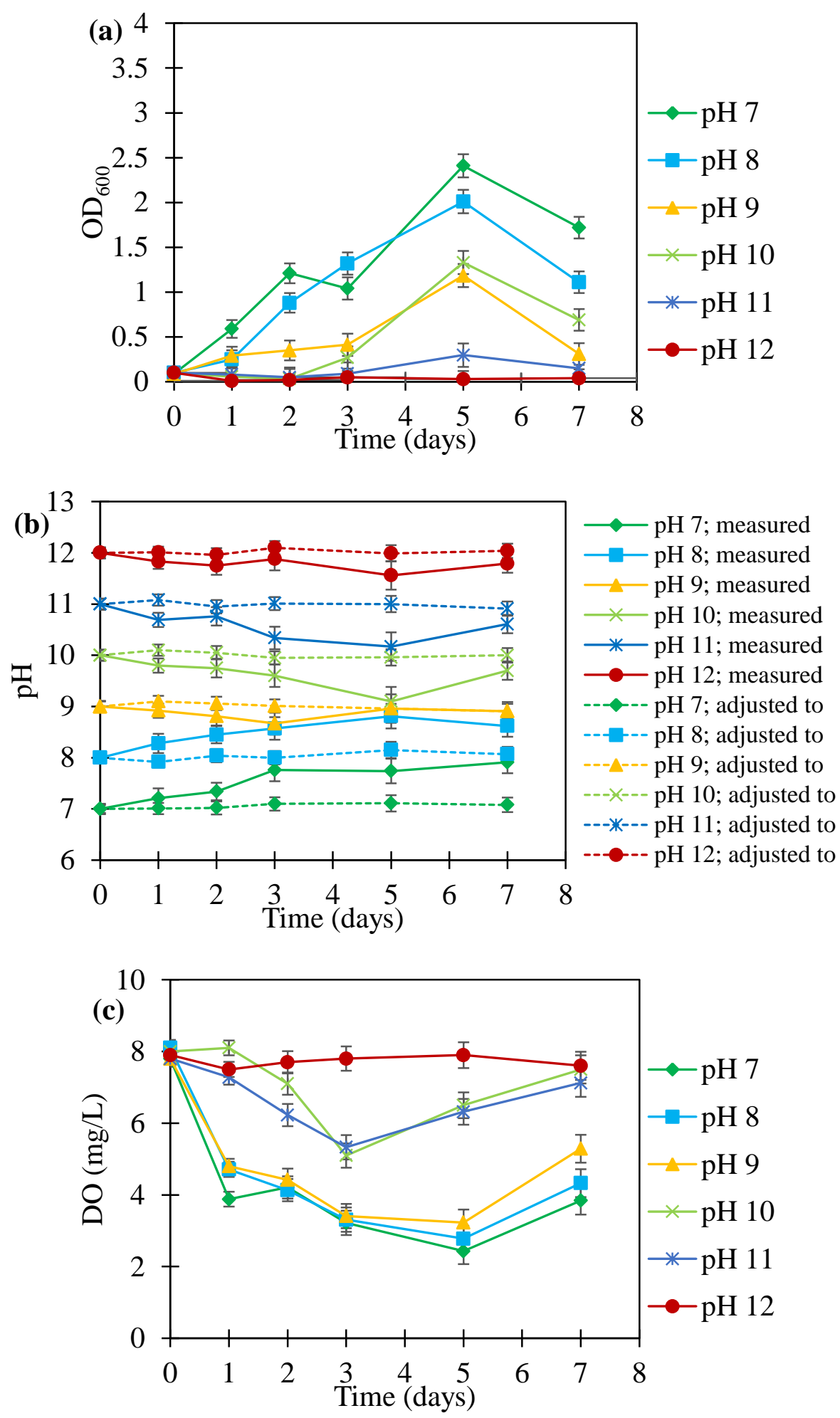

Figure S2. Changes in (a) $\mathrm{OD}_{600}$, (b) $\mathrm{pH}$, and (c) DO over time monitoring growth of bacteria B. megaterium situated in modified LB medium at $\mathrm{pH}$ controlled medium ranging from 7 to 12 . 

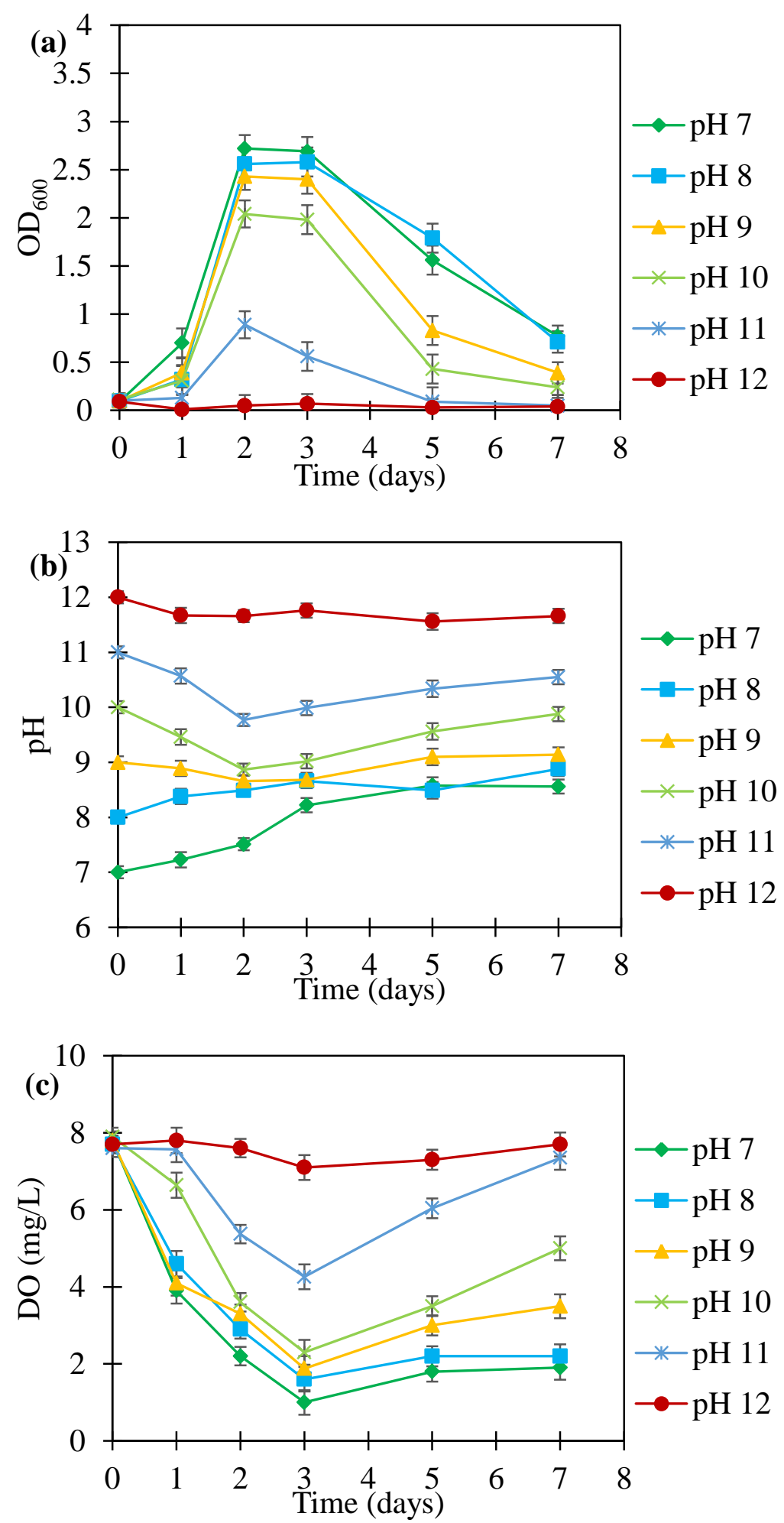

Figure $\mathrm{S} 3$. Changes in (a) $\mathrm{OD}_{600}$, (b) $\mathrm{pH}$, and (c) $\mathrm{DO}$ over time for bio-CN production by bacteria B. megaterium situated in modified LB medium containing $5 \mathrm{~g} / \mathrm{L}$ glycine at initial $\mathrm{pH}$ of 7 to 12 (no $\mathrm{pH}$ control). 

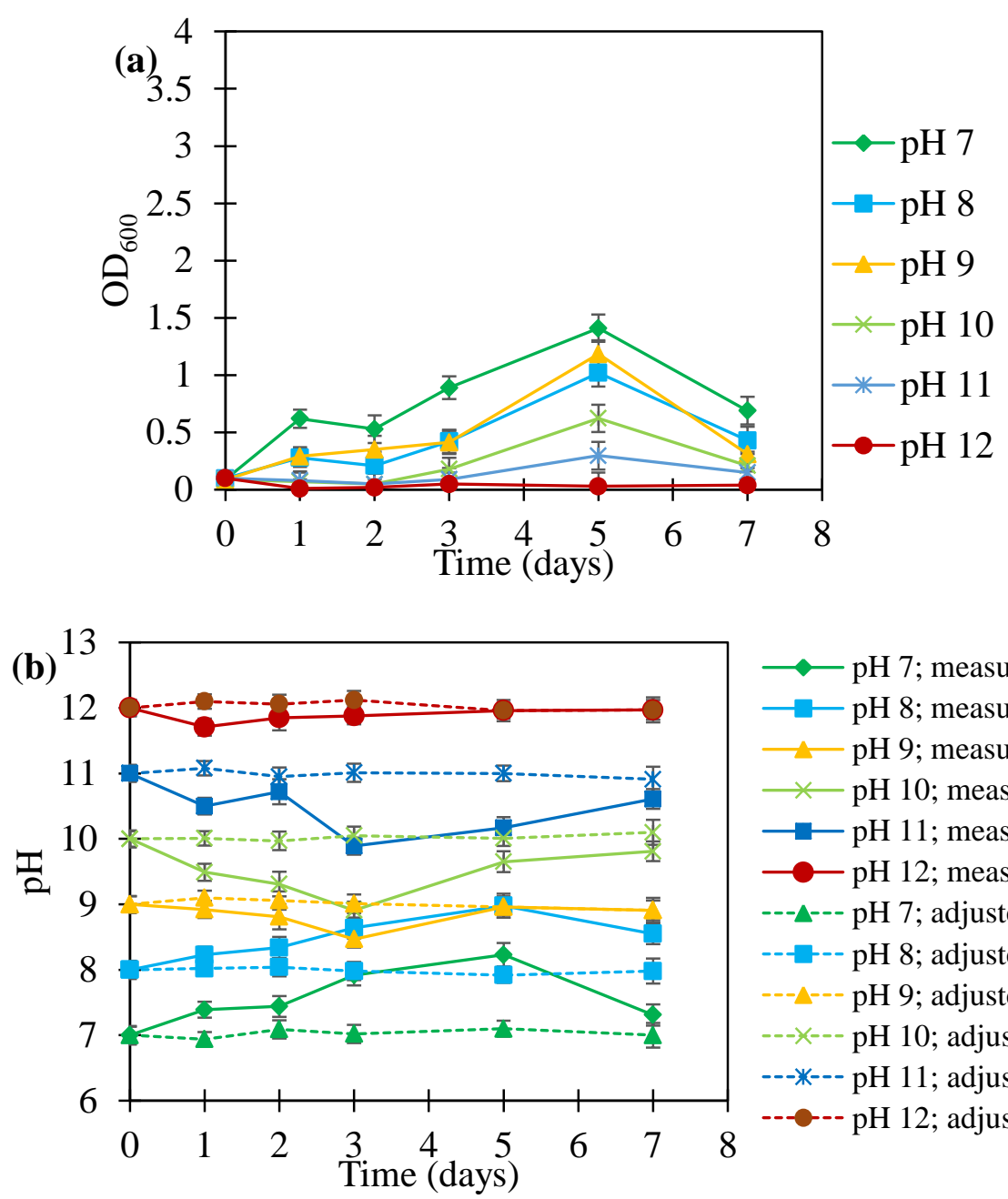

$\longrightarrow \mathrm{pH} 7$; measured

- $\mathrm{pH}$ 8; measured

$\longrightarrow \mathrm{pH} 9$; measured

$\times \quad \mathrm{pH} 10$; measured

$\longrightarrow$ - $\mathrm{pH} 11$; measured

- $\mathrm{pH} 12$; measured

---А--- pH 7; adjusted to

--- -- pH 8; adjusted to

----- pH 9; adjusted to

---×--- pH 10; adjusted to

---*--- pH 11; adjusted to

------ pH 12; adjusted to

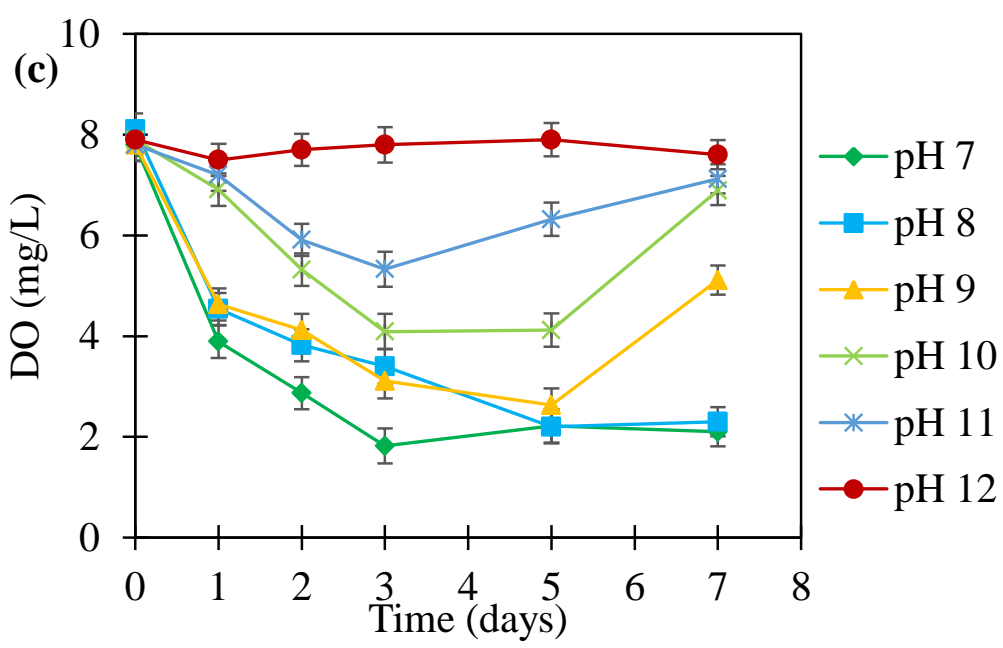

Figure $\mathrm{S} 4$. Changes in (a) $\mathrm{OD}_{600}$, (b) $\mathrm{pH}$, and (c) $\mathrm{DO}$ over time for bio-CN production by bacteria B. megaterium situated in modified LB medium containing $5 \mathrm{~g} / \mathrm{L}$ glycine at $\mathrm{pH}$ controlled medium ranging from 7 to 12. 


\section{Green chemistry metrics:}

\section{Ore 01:}

Table S1. Quantities Required for Green Chemistry Metrics Calculation for Biocyanidation of Ore O1

\begin{tabular}{|c|c|c|c|c|c|c|}
\hline \multirow{2}{*}{ Type } & Name & $\begin{array}{c}\text { Molar } \\
\text { mass } \\
(\mathrm{g} / \mathrm{mole})\end{array}$ & $\begin{array}{c}\text { Wt.\% or } \\
\text { concentration }\end{array}$ & Mass (g) & $\begin{array}{c}\text { Volume } \\
(\mathrm{mL})\end{array}$ & Moles \\
\hline Solvent & $\mathrm{H}_{2} \mathrm{O}$ & 18 & & 100 & 100 & 5.56 \\
\hline \multirow{5}{*}{ Reactant } & $\mathrm{C}_{2} \mathrm{H}_{5} \mathrm{NO}_{2}($ Glycine $)$ & 75 & $5(\mathrm{~g} / \mathrm{L})$ & 0.50 & & $6.67 * 10^{-3}$ \\
\cline { 2 - 7 } & $\mathrm{CN}^{-}$ & 26 & & $6.20^{*} 10^{-3}$ & & $2.38^{*} 10^{-4}$ \\
\cline { 2 - 7 } & $\mathrm{O}_{2}{ }^{\mathrm{a}}$ & 32 & & $4.00^{*} 10^{-4}$ & & $1.25^{*} 10^{-5}$ \\
\cline { 2 - 7 } Product & $\mathrm{Au}$ & 249 & & $2.70^{*} 10^{-5}$ & & $1.37^{*} 10^{-7}$ \\
\cline { 2 - 7 } & $\mathrm{Au}(\mathrm{CN})_{2}^{-}$ & & & $2.98^{*} 10^{-5}$ & & $1.20^{*} 10^{-7}$ \\
\hline Waste & Gold bearing & & & 100 & & \\
\hline
\end{tabular}

${ }^{a}$ DO stayed greater than $4 \mathrm{mg} / \mathrm{L}$ over the whole process for the optimum leaching condition

Table S2. Output of Bio-cyanidation of Ore O1

\begin{tabular}{|c|c|c|c|c|c|}
\hline \multirow{3}{*}{ Output } & Product & $\begin{array}{c}\text { Molar mass } \\
(\mathrm{g} / \text { mole })\end{array}$ & Mass $(\mathrm{g})$ & Moles & Yield (\%) \\
\cline { 2 - 6 } & $A u(C N)_{2}^{-}$ & 249 & $2.98 * 10^{-5}$ & $1.20^{*} 10^{-7}$ & 87.32 \\
\hline
\end{tabular}

Table S3. Calculated Green Chemistry Metrics for Bio-cyanidation of Ore O1

\begin{tabular}{|c|c|}
\hline Metrics & Calculations \\
\hline Percentage yield (\%) & 87.32 \\
\hline Percentage conversion (\%) & 87.32 \\
\hline Selectivity & 1.00 \\
\hline Stoichiometric factor & 1.999 \\
\hline Atom economy (\%) & 91.54 \\
\hline Reaction mass efficiency (\%) & 62.66 \\
\hline Optimum efficiency (\%) & 68.45 \\
\hline Process mass intensity & 208.96 \\
\hline
\end{tabular}




\section{Ore 02:}

Table S4. Quantities Required for Green Chemistry Metrics Calculation for Biocyanidation of Ore O2

\begin{tabular}{|c|c|c|c|c|c|c|}
\hline \multirow{2}{*}{ Type } & Name & $\begin{array}{c}\text { Molar } \\
\text { mass } \\
(\mathrm{g} / \mathrm{mole})\end{array}$ & $\begin{array}{c}\text { Wt.\% or } \\
\text { concentration }\end{array}$ & Mass (g) & $\begin{array}{c}\text { Volume } \\
(\mathrm{mL})\end{array}$ & Moles \\
\hline Solvent & $\mathrm{H}_{2} \mathrm{O}$ & 18 & & 100 & 100 & 5.55 \\
\hline & $\mathrm{C}_{2} \mathrm{H}_{5} \mathrm{NO}_{2}(\mathrm{Glycine})$ & 75 & $5(\mathrm{~g} / \mathrm{L})$ & 0.50 & & $6.67 * 10^{-3}$ \\
\hline \multirow{3}{*}{ Reactant } & $\mathrm{CN}^{-}$ & 26 & & $6.20^{*} 10^{-3}$ & & $2.38^{*} 10^{-4}$ \\
\cline { 2 - 7 } & $\mathrm{O}_{2}^{\mathrm{a}}$ & 32 & & $4.00^{*} 10^{-4}$ & & $1.25^{*} 10^{-5}$ \\
\cline { 2 - 7 } & $\mathrm{Au}$ & 197 & & $8.75^{*} 10^{-5}$ & & $4.44^{*} 10^{-7}$ \\
\hline \multirow{3}{*}{ Product } & $\mathrm{Au}(\mathrm{CN})_{2}^{-}$ & 249 & & $4.69 * 10^{-5}$ & & $1.88^{*} 10^{-7}$ \\
\cline { 2 - 7 } & $\begin{array}{c}\text { Gold bearing } \\
\text { solution }\end{array}$ & & & 100 & & \\
\hline Waste & Residue & & & 9.29 & & \\
\hline
\end{tabular}

${ }^{a}$ DO stayed greater than $4 \mathrm{mg} / \mathrm{L}$ over the whole process for the optimum leaching condition

Table S5. Output of bio-cyanidation of ore $\mathrm{O} 2$

\begin{tabular}{|c|c|c|c|c|c|}
\hline \multirow{2}{*}{ Output } & Product & $\begin{array}{c}\text { Molar mass } \\
(\mathrm{g} / \text { mole })\end{array}$ & Mass (g) & Moles & Yield (\%) \\
\cline { 2 - 6 } & $A u(C N)_{2}^{-}$ & 249 & $4.69 * 10^{-5}$ & $1.88 * 10^{-7}$ & 42.41 \\
\hline
\end{tabular}

Table S6. Calculated green chemistry metrics for bio-cyanidation of ore $\mathrm{O} 2$

\begin{tabular}{|c|c|}
\hline Metrics & Calculations \\
\hline Percentage yield (\%) & 42.41 \\
\hline Percentage conversion (\%) & 42.41 \\
\hline Selectivity & 1.00 \\
\hline Stoichiometric factor & 1.998 \\
\hline Atom economy (\%) & 91.54 \\
\hline Reaction mass efficiency (\%) & 30.43 \\
\hline Optimum efficiency (\%) & 33.24 \\
\hline Process mass intensity & 134.06 \\
\hline
\end{tabular}




\section{Green chemistry metrics calculations:}

$\mathrm{C}_{2} \mathrm{H}_{5} \mathrm{NO}_{2}$ (Glycine) $\stackrel{-2 \mathrm{H}^{+}}{\longrightarrow} \mathrm{C}_{2} \mathrm{H}_{3} \mathrm{NO}_{2}$ (aminoacetic acid) $\stackrel{-2 \mathrm{H}^{+}}{\longrightarrow} \mathrm{HCN}+\mathrm{CO}_{2}$

$4 \mathrm{Au}+8 \mathrm{CN}^{-}+\mathrm{O}_{2}+2 \mathrm{H}_{2} \mathrm{O} \rightarrow 4 \mathrm{Au}(\mathrm{CN})_{2}^{-}+4 \mathrm{OH}^{-}$

1. Percentage yield

Percentage yield $=\frac{\text { moles of product }}{\text { moles of limiting reactant }} \times 100$

For ore $\mathrm{O} 1: \frac{1.20 \times 10^{-7}}{1.37 \times 10^{-7}} \times 100=87.32 \%$

For ore $\mathrm{O} 2: \frac{1.88 \times 10^{-7}}{4.44 \times 10^{-7}} \times 100=42.41 \%$

2. Percentage conversion

Percentage conversion $=\left(1-\frac{\text { final mass of limiting reactant }}{\text { initial mass of limiting reactant }}\right) \times 100$

For ore O1: $\left(1-\frac{2.70 \times 10^{-5}-\left(197 \times 1.20 \times 10^{-7}\right)}{2.70 \times 10^{-5}}\right) \times 100=87.32 \%$

For ore $\mathrm{O} 2:\left(1-\frac{8.75 \times 10^{-5}-\left(197 \times 1.88 \times 10^{-7}\right)}{8.75 \times 10^{-5}}\right) \times 100=42.41 \%$

3. Selectivity

Selectivity $=\frac{\text { yield }}{\text { conversion }}$

For ore $\mathrm{O} 1: \frac{87.32}{87.32}=1.00$

For ore $\mathrm{O} 2: \frac{42.41}{42.41}=1.00$

4. Stoichiometric factor (SF)

$S F=1+\frac{\text { total masses of extra reagents }}{\text { stoichiometric masses of all reagents }}$ 
For ore O1: $1+\frac{26 \times\left(2.38 \times 10^{-4}-2 \times 1.20 \times 10^{-7}\right)}{6.20 \times 10^{-3}}=1.999$

For ore O2: $1+\frac{26 \times\left(2.38 \times 10^{-4}-2 \times 1.88 \times 10^{-7}\right)}{6.20 \times 10^{-3}}=1.998$

\section{Atom economy (AE)}

$A E=\frac{\text { molar mass of product }}{\text { total molar mass of reactants }} \times 100$

For ore $\mathrm{O} 1: \frac{249}{75+197} \times 100=91.54 \%$

For ore O2: $\frac{249}{75+197} \times 100=91.54 \%$

6. Reaction mass efficiency (RME)

$R M E=\frac{\text { molar mass of product }}{\sum(\text { molar mass of reactant } i \times \text { molar ration of reactant } i \text { to ractant } 1)} \times$ yield

For ore O1: $\frac{249}{197+75 \times 2 / 1} \times 87.32=62.66 \%$

For ore O2: $\frac{249}{197+75 \times 2 / 1} \times 42.41=30.43 \%$

7. Optimum efficiency (OE)

$O E=\frac{R M E}{A E} \times 100$

For ore O1: $\frac{62.66}{91.54} \times 100=68.45 \%$

For ore O2: $\frac{30.43}{91.54} \times 100=33.24 \%$

8. Process mass intensity (PMI)

$P M I=\frac{\text { total mass used in the process }}{\text { mass of the product }}$

For ore O1: $\frac{6.20 \times 10^{-3}+2.70 \times 10^{-5}}{2.98 \times 10^{-5}}=208.96$ 
For ore $\mathrm{O} 2: \frac{6.20 \times 10^{-3}+4.69 \times 10^{-5}}{8.75 \times 10^{-5}}=134.06$ 